\title{
ON THE ESSENTIAL DIMENSION OF INFINITESIMAL GROUP SCHEMES
}

\author{
DAJANO TOSSICI AND ANGELO VISTOLI
}

\begin{abstract}
We discuss essential dimension of group schemes, with particular attention to infinitesimal group schemes. We prove that the essential dimension of a group scheme of finite type over a field $k$ is greater than or equal to the difference between the dimension of its Lie algebra and its dimension. Furthermore, we show that the essential dimension of a trigonalizable group scheme of length $p^{n}$ over a field of characteristic $p>0$ is at most $n$. We give several examples.
\end{abstract}

\section{INTRODUCTION}

The notion of essential dimension of a finite group over a field $k$ was introduced by Buhler and Reichstein ([BR97]). It was later extended to various contexts. First Reichstein generalized it to linear algebraic groups ([Rei00]) in characteristic zero; afterwards Merkurjev gave a general definition for covariant functors from the category of extension fields of the base field $k$ to the category of sets ([BF03]). Brosnan, Reichstein and Vistoli ([BRV09]) studied the essential dimension of algebraic stacks, a general class which includes almost all the examples of interest.

Important results on the essential dimension of finite groups in characteristic 0 have been proved by Florence ([Flo07]) and Karpenko and Merkurjev ([KM08]). The essential dimension of finite groups in positive characteristic has been studied by Ledet [Led04]. As to higher dimensional groups, there are works of Reichstein and Youssin ([RY00]), Chernousov and Serre ([CS06]), Gille and Reichstein [GR09], Brosnan, Reichstein and Vistoli [BRV10] on algebraic groups, Brosnan [Bro07] on abelian varieties over $\mathbb{C}$, and Brosnan and Shreekantan [BS08] on abelian varieties over number fields. The case of non-smooth group schemes (necessarily in positive characteristic) has not been investigated.

Let $k$ be a field of characteristic $p>0$. For any scheme $X$ over $k$ let $X^{(p)}$ denote the scheme $X \times_{\text {Spec } k}$ Spec $k$, where Spec $k$ is endowed with the structure of $k$-scheme via the absolute Frobenius, which at the level of algebras is given by $a \mapsto a^{p}$. We will endow $X^{(p)}$ with the structure of a $k$-scheme given by the projection on the second factor. Moreover we consider the relative Frobenius $\mathrm{F}: X \mapsto X^{(p)}$, which is the $k$-morphism induced by the absolute Frobenius on $X$. We observe that if the $k$-scheme $X$ is in fact defined over $\mathbb{F}_{p}$ then $X^{(p)}$ is isomorphic to $X$. When this is the case we replace $X^{(p)}$ with $X$.

If $m$ is a positive integer, we denote as usual by $\boldsymbol{\alpha}_{p^{m}}$ the kernel of the $m$-th power of the relative Frobenius $F^{m}: \mathbb{G}_{\mathrm{a}} \rightarrow \mathbb{G}_{\mathrm{a}}$ over $k$, while $\boldsymbol{\mu}_{p^{m}}$ is the kernel of the $p^{m \text { th }}$-power homomorphism $\mathbb{G}_{\mathrm{m}} \rightarrow \mathbb{G}_{\mathrm{m}}$. It is certainly known to experts that the essential dimensions of $\boldsymbol{\alpha}_{p^{m}}$ and $\boldsymbol{\mu}_{p^{m}}$ are 1, and that the essential dimension

Date: September 4, 2010. 
of $\boldsymbol{\mu}_{p^{m}}^{n}$ is $n$. To the authors' knowledge nothing else was known. The purpose of this paper is to throw some light on this subject. In particular we will focus on the essential dimension of infinitesimal (i.e. connected and finite) group schemes. This could give, for instance, some information about the essential dimension of Abelian varieties over a field $k$ of characteristic $p>0$ with $p$-rank equal to zero. The $p$-rank $f$ of an Abelian variety $A$ is defined by $A[p](\bar{k}) \simeq(\mathbb{Z} / p \mathbb{Z})^{f}$, where $A[p]$ is the group scheme of $p$-torsion points and $\bar{k}$ is an algebraic closure of $k$. If an Abelian variety $A$ has positive $p$-rank, its essential dimension is conjecturally infinite, since for any $n>0, A$ contains, over $\bar{k}$, the group scheme $\mathbb{Z} / p^{n} \mathbb{Z}$, whose essential dimension is conjecturally equal to $n$ (see Lemma 2.1 and Example 4.9).

If $G$ is a group scheme of finite type over a field $k$, its essential $\operatorname{dimension~} \operatorname{ed}_{k} G$ is the essential dimension of the stack $\mathcal{B}_{k} G$. Let us recall the definition. Let $k$ be a field, and $G$ be a group scheme of finite type over $k$. If $X$ is a an algebraic space, over $k$, a $G$-torsor on $X$ is an algebraic space $P$ over $k$ with a right action of $G$, with a $G$-invariant morphism $P \rightarrow X$, such that fppf locally on $X$ the scheme $P$ is $G$-equivariantly isomorphic to $X \times_{\operatorname{Spec} k} G$. We recall that if $X$ is a scheme and $G$ is affine then any $G$-torsor over $X$ is in fact a scheme ([Mil80, Theorem III $4.3])$. Isomorphism classes of $G$-torsors on $X$ form a pointed set $\mathrm{H}^{1}(X, G)$; if $G$ is commutative, then $\mathrm{H}^{1}(X, G)$ is a group, and coincides with the cohomology group of $G$ in the fppf topology.

Definition 1.1. Let $G$ be a group scheme of finite type over a field $k$. Let $k \subseteq K$ be an extension field and $[\xi] \in \mathrm{H}^{1}(\operatorname{Spec}(K), G)$ the class of a $G$-torsor $\xi$. Then the essential dimension of $\xi$ over $k$, which we denote by $\operatorname{ed}_{k} \xi$, is the smallest nonnegative integer $n$ such that there exists a subfield $L$ of $K$ containing $k$, with $\operatorname{tr} \operatorname{deg}(L / k) \leq n$ such that $[\xi]$ is in the image of the morphism $\mathrm{H}^{1}(\operatorname{Spec}(L), G) \rightarrow \mathrm{H}^{1}(\operatorname{Spec}(K), G)$.

The essential dimension of $G$ over $k$, which we denote by $\operatorname{ed}_{k} G$, is the supremum of ed $k \xi$, where $K / k$ ranges through all the extension of $K$, and $\xi$ ranges through all the $G$-torsors over $\operatorname{Spec}(K)$.

The essential dimension of $\xi$ at a prime integer $p$, which we denote by $\operatorname{ed}_{k}(\xi ; p)$, is the minimal value of $\operatorname{ed}_{k}\left(\xi_{K^{\prime}}\right)$, as $K^{\prime}$ ranges over all finite field extensions $K^{\prime} / K$ such that $p$ does not divide the degree $\left[K^{\prime}: K\right]$ and $\left[\xi_{K^{\prime}}\right]$ is the image of $[\xi]$ in $\mathrm{H}^{1}\left(\operatorname{Spec}\left(K^{\prime}\right), G\right)$. Finally the essential dimension $\operatorname{ed}_{k}(G ; p)$ is defined as the supremum value of $\operatorname{ed}_{k}(\xi ; p)$, as $K$ ranges over all field extensions of $k$ and $\xi$ ranges over $\mathrm{H}^{1}(\operatorname{Spec}(K), G)$.

It is clear from definition that $\operatorname{ed}_{k} G \geq \operatorname{ed}_{k}(G ; p)$ for any integer $p$. If $G$ is smooth, then $G$-torsors are locally trivial in the étale topology, and our definition coincides with that of Berhuy and Favi ([BF03]); in the general case, to get meaningful results one needs to use the fppf topology. For example, if $G$ is an infinitesimal group scheme, the $G$-torsors over a reduced scheme that are locally trivial in the étale topology are in fact trivial.

Our first result is a general lower bound for the essential dimension.

Theorem 1.2. Let $G$ be a group scheme of finite type over a field $k$ of characteristic $p \geq 0$. Then

$$
\operatorname{ed}_{k}(G ; p) \geq \operatorname{dim}_{k} \operatorname{Lie} G-\operatorname{dim} G .
$$

We also have a fairly general upper bound. Let us recall the definition of a trigonalizable group scheme. 
Definition 1.3. Let $G$ be an affine group scheme of finite type over a field $k$. We say that $G$ is trigonalizable if it has a normal unipotent subgroup scheme $U$ such that $G / U$ is diagonalizable.

The name is justified by the fact that, every trigonalizable group scheme over $k$, is a subgroup scheme of the group scheme of upper triangular $n \times n$ matrices over $k$, for some $n$. Any affine commutative group scheme over an algebraically closed field is trigonalizable (see [DG70, Theorem IV $\S 3,1.1]$ ).

Theorem 1.4. Let $G$ be a finite trigonalizable group scheme over a field of characteristic $p>0$, of order $p^{n}$. Then $\operatorname{ed}_{k} G \leq n$.

For constant $p$-group schemes (which are unipotent) the above result has already been proved by Ledet ([Led04]).

The second and the third sections are devoted to the proofs of these two theorems. In the last section, combining the lower and upper bounds above, we calculate the essential dimension of some classes of infinitesimal group schemes. In particular we prove that the essential dimension of a trigonalizable group scheme of height $\leq 1$, i.e. such that the Frobenius $\mathrm{F}$ is trivial on it, is equal to the dimension of its Lie algebra (Corollary 4.5). Example 4.1, due to R. Lötscher, M. MacDonald, A. Meyer and Z. Reichstein, shows that there exist infinitesimal group schemes with essential dimension strictly larger than the dimension of its Lie algebra. However, we don't have similar examples over an algebraically closed field. In Example 4.8 we propose a class of a commutative unipotent group scheme whose essential dimensions we are unable to determine, which should be an important test case to determine whether it is reasonable to conjecture that equality holds for trigonalizable group schemes.

Acknowledgements. We would like to thank P. Brosnan, F. Oort and Z. Reichstein for useful comments and corrections to the first version of this paper. We are also in debt with the referee for his very detailed and useful report.

\section{The Proof of Theorem 1.2}

First we first state a well known fact.

Lemma 2.1 ([Mer09, Corollary 4.3]). If $G$ is a subgroup scheme of a group scheme $H$, then

(a) $\operatorname{ed}_{k} G+\operatorname{dim} G \leq \operatorname{ed}_{k} H+\operatorname{dim} H$, and

(b) $\operatorname{ed}_{k}(G ; p)+\operatorname{dim} G \leq \operatorname{ed}_{k}(H ; p)+\operatorname{dim} H$ for any prime $p$.

Now we prove the Theorem. If the characteristic of $k$ is 0 , then $G$ is smooth and there is nothing to prove. Suppose that the characteristic of $k$ is $p>0$. Since the essential dimension does not increase after a base change ([BF03, Proposition 1.5]), we may assume that $k$ is algebraically closed.

Let $G_{1}$ be the kernel of the relative Frobenius map $\mathrm{F}: G \rightarrow G^{(p)}$; then $G_{1}$ is an infinitesimal group scheme, and $\operatorname{Lie} G_{1}=\operatorname{Lie} G$. Since by Lemma 2.1 we have $\operatorname{ed}_{k}\left(G_{1} ; p\right) \leq \operatorname{ed}_{k}(G ; p)+\operatorname{dim} G$, it is sufficient to show that $\operatorname{ed}_{k}\left(G_{1} ; p\right) \geq$ $\operatorname{dim}_{k} \operatorname{Lie} G_{1}$; in other words, we may assume that $G=G_{1}$, i.e., by definition, $G$ has height at most 1 .

Let $G$ act freely on an open subscheme $X$ of a representation of $G$. If $K$ is the function field of the quotient $X / G$ and $E$ is the function field of $X$, then we have a $G$-torsor $\operatorname{Spec} E \rightarrow \operatorname{Spec} K$. Set $n \stackrel{\text { def }}{=} \operatorname{dim}_{k} \operatorname{Lie} G$. Let $K^{\prime} / K$ be any extension 
of degree coprime to $p$. We need to prove that for any such $K^{\prime}$ then the essential dimension of the $G$-torsor $\operatorname{Spec}\left(E \otimes_{K} K^{\prime}\right) \rightarrow \operatorname{Spec} K^{\prime}$ is at least $\operatorname{dim}_{k} \operatorname{Lie} G$. Since the extension $K^{\prime} / K$ is separable, while $E / K$ is purely inseparable, we have that $E \otimes_{K} K^{\prime}$ is again a field. Suppose that the $G$-torsor is defined over an extension $L$ of $k$ contained in $K^{\prime}$; we need to show that the transcendence degree of $L$ over $k$ is at least $n$. Let $\operatorname{Spec} R \rightarrow \operatorname{Spec} L$ be the $G$-torsor yielding $\operatorname{Spec}\left(E \otimes_{K} K^{\prime}\right) \rightarrow \operatorname{Spec} K^{\prime}$ by base change. Clearly $R$ is a field. By the definition of the Frobenius we have that the diagram

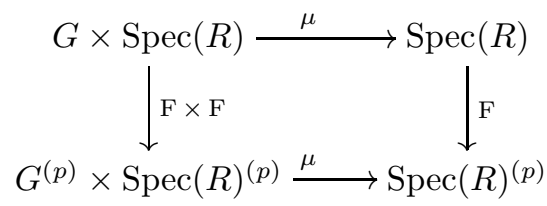

commutes, where $\mu$ is the action of $G$ on $\operatorname{Spec}(R)$. Since $\mathrm{F}$ is trivial on $G$ it follows, by the above diagram, that the Frobenius $\mathrm{F}: \operatorname{Spec}(R) \rightarrow \operatorname{Spec}(R)^{(p)}$ is $G$-equivariant, where we consider the trivial action on the target. Therefore $\mathrm{F}$ : $\operatorname{Spec}(R) \mapsto \operatorname{Spec}(R)^{(p)}$ factorizes through $\operatorname{Spec}(R) \rightarrow \operatorname{Spec}(R) / G=\operatorname{Spec}(L)$. But the degree of the Frobenius $\mathrm{F}: \operatorname{Spec}(R) \rightarrow \operatorname{Spec}(R)^{(p)}$ is equal, by the next lemma, to $p^{d}$, where $d \stackrel{\text { def }}{=} \operatorname{tr} \operatorname{deg}_{k} R$. While the degree of $\operatorname{Spec}(R) \rightarrow \operatorname{Spec}(L)$ is equal to the order of $G$, which is $p^{n}$ with $n=\operatorname{dim}_{k}(\operatorname{Lie} G)$. Hence we have $\operatorname{tr} \operatorname{deg}_{k} R=$ $\operatorname{tr} \operatorname{deg}_{k} L \geq \operatorname{dim}_{k}(\operatorname{Lie} G)$, as wanted.

Lemma 2.2. [Liu02, Corollary 3.2.27] Let $R$ be a finitely generated extension of transcendence degree $d$ of a perfect field $k$ of characteristic $p>0$. Then the relative Frobenius $\mathrm{F}: \operatorname{Spec}(R) \rightarrow \operatorname{Spec}(R)^{(p)}$ is a finite morphism of degree $p^{d}$.

\section{The PRoof of Theorem 1.4}

Let us start with stating a few Lemmas that we will use in the proof. The first two are well known.

Lemma 3.1. [DG70, Proosition IV $\S 2,2.5]$ Let $G$ be a commutative unipotent group scheme over a field $K$. Then there exists a central decomposition series

$$
1=G_{0} \subseteq G_{1} \subseteq \cdots \subseteq G_{r}=G
$$

of $G$, such that each successive quotient $G_{i} / G_{i-1}$ is a subgroup scheme of $\mathbb{G}_{\mathrm{a}}$.

Lemma 3.2. [DG70, Corollary IV §2, 2.6] If $G$ is a group scheme over a field $K$ and $E$ is an extension of $K$, then $\operatorname{Spec} E \times \operatorname{Spec} K G \rightarrow \operatorname{Spec} E$ is unipotent if and only if $G$ is unipotent. In particular, any twisted form of a unipotent group scheme is unipotent.

Lemma 3.3. Let $G$ be a commutative unipotent group scheme over a field $K$. Then $\mathrm{H}^{i}(K, G)=0$, for $i \geq 2$.

Proof. By Lemma 3.1, we may assume that $G$ is a subgroup of $\mathbb{G}_{\mathrm{a}}$. The quotient $\mathbb{G}_{\mathrm{a}} / G$ is isomorphic to $\mathbb{G}_{\mathrm{a}}$ ([DG70, Proposition IV $\left.\left.\S 2,1.1\right]\right)$; then the result follows from the fact that $\mathrm{H}^{i}\left(K, \mathbb{G}_{\mathrm{a}}\right)=0$ for $i \geq 1$.

Now we prove the key Lemma. 
Lemma 3.4. Suppose that we have an extension

$$
1 \longrightarrow G_{1} \longrightarrow G \longrightarrow G_{2} \longrightarrow 1
$$

of group schemes over $k$, where $G_{1}$ is a commutative unipotent normal group subscheme of a group scheme $G$. Let $P \rightarrow \operatorname{Spec} K$ be a $G$-torsor over an extension $K$ of $k$. Then there exists an intermediate extension $k \subseteq F \subseteq K$ and a twisted form $\widetilde{G_{1}} \rightarrow \operatorname{Spec} F$ of $G_{1}$ over $F$, such that $P$ is defined over an intermediate extension of transcendence degree at most $\operatorname{ed}_{k} G_{2}+\operatorname{ed}_{F} \widetilde{G_{1}}$ over $k$.

Furthermore, if $G_{1}$ is central in $G$, then $\widetilde{G_{1}}=\operatorname{Spec} F \times_{\operatorname{Spec} k} G_{1}$.

Proof. Consider the induced $G_{2}$-torsor $Q \stackrel{\text { def }}{=} P / G_{1} \rightarrow$ Spec $K$. There exists an intermediate extension $k \subseteq E \subseteq K$ with $\operatorname{tr} \operatorname{deg}_{k} E \leq \operatorname{ed} G_{2}$, such that $Q$ comes by base change from a $G_{2}$-torsor $Q_{E} \rightarrow \operatorname{Spec} E$. I claim that $Q_{E}$ lifts to a $G$-torsor $P_{E} \rightarrow$ Spec $E$.

To see this, consider the fppf gerbe of liftings $\mathcal{L} \rightarrow(\mathrm{Sch} / E)$. It is a fibered category over the category ( $\mathrm{Sch} / E$ ) of $E$-schemes, whose objects over an $E$-scheme $T \rightarrow$ Spec $E$ are $G$-torsors $P_{T} \rightarrow T$, together with isomorphisms of $G_{2}$-torsors $P_{T} / G_{1} \simeq T \times_{\text {Spec } E} Q_{E}$, or, equivalently, $G$-equivariant morphisms of $T$-schemes $P_{T} \rightarrow T \times_{\text {Spec } E} Q_{E}$. The arrows from $P_{T} \rightarrow T \times_{\operatorname{Spec} E} Q_{E}$ to $P_{T^{\prime}}^{\prime} \rightarrow T^{\prime} \times_{\operatorname{Spec} E} Q_{E}$ are defined in the obvious way, as diagrams

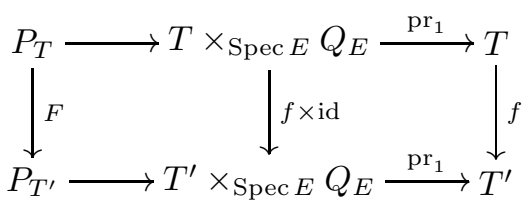

in which $F$ is $G$-equivariant. We need to show that $\mathcal{L}$ has a global section over Spec E.

The action of $G$ on $G_{1}$ by conjugation descends to an action of $G_{2}$ on $G_{1}$, since $G_{1}$ is commutative. Denote by $\widetilde{G_{1}}$ the twisted form of the group scheme Spec $E \times \times_{\text {Spec } k} G_{1}$ coming from the $G_{2}$-torsor $Q_{E} \rightarrow$ Spec $E$; in other words, $\widetilde{G_{1}}$ is the quotient $\left(Q_{E} \times_{\text {Spec } k} G_{1}\right) / G_{2}$, where $G_{2}$ acts on the right on $Q_{E}$, and on $G_{1}$ by left conjugation. We claim that the gerbe $\mathcal{L}$ is banded by $\widetilde{G_{1}}$; that is, if $P_{T} \rightarrow T \times{ }_{\text {Spec } E} Q_{E}$ is an object of $\mathcal{L}(T)$, the automorphism group $\operatorname{Aut}\left(P_{T}\right)$ is isomorphic to $\widetilde{G_{1}}(T)$, and this isomorphism is functorial in $T$. In fact, the twisted form $\widetilde{G}$ of $G$ obtained as the quotient $P_{T} \times_{\text {Spec } k} G$ by the diagonal action of $G$ (where $G$ acts on itself by conjugation) is the automorphism group scheme of the $G$-torsor $P_{T} \rightarrow T$, and it contains $\widetilde{G_{1}}$ as the subgroup scheme of automorphisms inducing the identity on $T \times{ }_{\operatorname{Spec}} Q_{E}$. Hence $\widetilde{G_{1}}$ is the automorphism group scheme of the object $P_{T} \rightarrow T \times_{\text {Spec } E} Q_{E}$, and this proves the claim.

By [Gir71, Section IV 3.4], the equivalence classes of gerbes banded by $\widetilde{G_{1}}$ are parametrized by the group $\mathrm{H}^{2}\left(K, \widetilde{G_{1}}\right)$; a gerbe corresponds to 0 , i.e., it is equivalent to the classifying stack $\mathcal{B}_{E} \widetilde{G_{1}}$, if and only if it has a section. Now, by Lemma 3.2 , $\widetilde{G_{1}}$ is unipotent; hence by Lemma $3.3 \mathrm{H}^{2}\left(E, \widetilde{G_{1}}\right)=0$, so $\mathcal{L}$ has a section, and the $G_{2}$-torsor $Q_{E} \rightarrow$ Spec $E$ lifts to a $G$-torsor $P_{E} \rightarrow Q_{E} \rightarrow \operatorname{Spec} E$.

There is no reason why $\operatorname{Spec} K \times_{\operatorname{Spec} E} P_{E}$ should be isomorphic to $P \rightarrow \operatorname{Spec} K$ as a $G$-torsor. However, by construction, we have $P / G_{1} \simeq \operatorname{Spec} K \times_{\operatorname{Spec} E} Q_{E}$. Since as we just saw $\mathcal{L}$ is a trivial gerbe banded by $\widetilde{G_{1}}$, we have that $\mathcal{L}$ is equivalent to the 
classifying stack $\mathcal{B}_{E} \widetilde{G_{1}}$, in such a way that the lifting $P_{E} \rightarrow Q_{E}$ corresponds to the trivial torsor $\widetilde{G_{1}} \rightarrow \operatorname{Spec} E$. Then $P \rightarrow Q$ gives an object of $\mathcal{L}(\operatorname{Spec} K)$; this will be defined over a intermediate extension $E \subseteq F \subseteq K$ of transcendence degree at $\operatorname{most}_{\operatorname{ed}} \widetilde{G_{1}}$ over $E$. So over $F$ there will exist an object $P_{F} \rightarrow \operatorname{Spec} F \times_{\operatorname{Spec} E} Q_{E}$ which is isomorphic to $P \rightarrow Q$ when pulled back to $K$. Hence $P$ is defined over $F$, and, since the transcendence degree of $F$ is at most equal to $\operatorname{ed}_{k} G_{2}+\operatorname{ed}_{E} \widetilde{G_{1}}$, the result follows.

Now we are ready to prove the theorem.

First of all, suppose that $G$ is a diagonalizable group of order $p^{n}$; then $G$ is a product $\boldsymbol{\mu}_{p^{d_{1}}} \times \cdots \times \boldsymbol{\mu}_{p^{d_{r}}}$ for certain positive integers $d_{1}, \ldots, d_{r}$ with $d_{1}+\cdots+d_{r}=n$. Then $G$ is a subgroup scheme of $\mathbb{G}_{\mathrm{m}}^{r}$, hence by the Lemma 2.1 we have ed $G \leq r \leq n$.

Now, assume that $G$ is commutative unipotent of order $p^{n}$, with $n>0$. Suppose that $G$ is a subgroup of $\mathbb{G}_{\mathrm{a}}$; then again by Lemma 2.1 we have ed ${ }_{k} G \leq \operatorname{dim} \mathbb{G}_{\mathrm{a}}=$ $1 \leq n$, and we are done.

If $G$ is not a subgroup scheme of $\mathbb{G}_{\mathrm{a}}$, we proceed by induction on $n$. Assume that the result holds for all commutative unipotent subgroup schemes of order $p^{m}$ with $m<n$. Let $G_{1}$ be a nontrivial subgroup scheme that is a subgroup scheme of $\mathbb{G}_{\mathrm{a}}$ and call $p^{m}$ its order. The group scheme $G_{1}$ exists by Lemma 3.1 . Then by Lemma 3.4 we have

$$
\operatorname{ed}_{k} G \leq \operatorname{ed}_{k}\left(G / G_{1}\right)+\operatorname{ed}_{F} G_{1 F} \leq(n-m)+m=n ;
$$

so the result holds for $G$.

Let $G$ be a trigonalizable infinitesimal group scheme of order $p^{n}$. Once again, we proceed by induction on $n$. Let us suppose that the result is true for all trigonalizable groups of order $p^{m}$ with $m<n$. By definition, $G$ is an extension

$$
1 \longrightarrow G_{\mathrm{u}} \longrightarrow G \longrightarrow G_{\mathrm{d}} \longrightarrow 1
$$

where $G_{\mathrm{u}}$ is unipotent and $G_{\mathrm{d}}$ is diagonalizable. We may assume that $G_{\mathrm{u}}$ is nontrivial, otherwise $G$ is diagonalizable and we are done. If $G_{1}$ denotes the center of $G_{\mathrm{u}}$, then $G_{1}$ is a nontrivial commutative unipotent normal subgroup of $G$; set $G_{2} \stackrel{\text { def }}{=} G / G_{1}$. Call $p^{m}$ the order of $G_{2}$; by induction hypothesis we have $\operatorname{ed}_{k} G_{2} \leq m$. Once again using Lemma 3.4, we have that $\operatorname{ed}_{k} G \leq \operatorname{ed}_{k} G_{2}+\operatorname{ed}_{F} \widetilde{G_{1}}$ for some twisted form of $G_{1}$; but by Lemma 3.2 the group scheme $\widetilde{G_{1}}$ is still commutative unipotent, hence by the previous case $\operatorname{ed}_{F} \widetilde{G_{1}} \leq n-m$, and we are done.

\section{The ESSENTIAL DIMENSION OF SOME GROUP SCHEMES}

The inequality of Theorem 1.2 is not an equality in general, even for infinitesimal group schemes. Furthermore, the inequality of Theorem 1.4 does not hold in general for finite non-trigonalizable group schemes.

Example 4.1. The following is due to R. Lötscher, M. MacDonald, A. Meyer and Z. Reichstein [LMMR09, Example 6.3]. Suppose that $\ell / k$ is a cyclic extension of degree $p$ of fields of characteristic $p>0$; call $\sigma$ a generator for the Galois group $\operatorname{Gal}(\ell / k)$. The automorphism group of $\boldsymbol{\mu}_{p^{2}}$ is a cyclic group of order $p(p-1)$; choosing an element of order $p$ in this automorphism group gives an action of $\operatorname{Gal}(\ell / k)$ on $\boldsymbol{\mu}_{p^{2}, \ell}$, which defines, by Galois descent, a form $G$ of $\boldsymbol{\mu}_{p^{2}}$ over $k$. This is an infinitesimal group scheme over $k$ of order $p^{2}$, and $\operatorname{dim}_{k} \operatorname{Lie} G=1$; however, its essential dimension is $p$. 
This example gives a strict inequality in Theorem 1.2, and shows how the inequality of Theorem 1.4 does not hold in general. However, we don't know any examples of either phenomenon over an algebraically closed field.

In order to investigate this question, let us give the following definition.

Definition 4.2. Let $G$ be an affine group scheme of finite type over a field $k$. If

$$
\operatorname{ed}_{k} G=\operatorname{dim}_{k} \operatorname{Lie}(G)-\operatorname{dim} G
$$

then $G$ is called almost special.

If $G$ is a linear smooth group scheme over a field $k$, then it is almost-special if and only if it has essential dimension 0. The term "almost-special" is justified by the following fact.

Recall that a linear group scheme $G$ over a field $k$ is called special if every $G$ torsor defined over an extension of $k$ is trivial. Special groups were introduced by J.-P. Serre in [Ser58] and studied by A. Grothendieck in [Gro58], and by many other authors since then.

Proposition 4.3. A smooth affine group scheme of finite type over a field is almostspecial if and only if it is special.

This is immediate over an algebraically closed field, but seems to be new in this generality; we are grateful to the referee and to Z. Reichstein (who also helped with the proof) for pointing this out.

Proof. Let $G$ be a smooth affine group of finite type over a field $k$. It is obvious that if $G$ is special, then it is almost-special.

Assume that $G$ is almost-special. Let $V$ be a generically free finite-dimensional representation of $G$; by definition, there exists an non-empty open subscheme $U$ on which the action is free. Let $U / G$ be the quotient, which exist as an integral algebraic space of finite type over $k$; then the projection $U \rightarrow U / G$ is a $G$-torsor. Let Spec $K$ be the generic point of $U / G$, and let $E \rightarrow$ Spec $K$ the pullback of the $G$-torsor $U \rightarrow U / G$. Since $\operatorname{ed}_{k} G=0$, then $E \rightarrow$ Spec $K$ is defined over an intermediate extension $k \subseteq \ell \subseteq K$ that is finite over $k$. Since $U / G$ is geometrically integral over $k$ we have that $k$ is algebraically closed in $K$, so $\ell=k$, and the torsor $E \rightarrow \operatorname{Spec} K$ is defined over $k$. We have a cartesian diagram

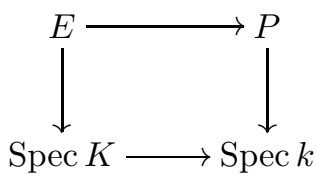

where $P \rightarrow$ Spec $k$ is $G$-torsor, and the morphism in the top row is $G$-equivariant.

Let us show that $G$ is connected. Let $G^{0}$ be the connected component of the identity in $G$. The scheme $P / G^{0}$ is integral, since $P$ is integral, and is finite over $k$; hence it is the spectrum of a finite extension of $k$ contained in $K$. So $P / G^{0}=\operatorname{Spec} k$, and $G=G^{0}$, as claimed.

If $k$ is finite, then it follows from a famous theorem of Steinberg [Ste65, Theorem 1.9] that every $G$-torsor over a finite field is trivial. Since $\operatorname{ed}_{k} G=0$ we see that every $G$-torsor over an extension of $k$ descends to a finite field, and so it is trivial.

Next, assume that $k$ is infinite. The torsor $E \rightarrow \operatorname{Spec} K$ is versal (see [GMS03, Example 5.3]), hence if we show that it is trivial, then every other torsor over 
an extension of $k$ will be trivial. The $G$-equivariant morphism $E \rightarrow P$ gives a $G$-equivariant rational map $U \rightarrow P$. After restricting $U$, we may assume that $U \rightarrow P$ is defined everywhere. Since $U$ is a non-empty open subset of an affine space over an infinite field, we have $U(k) \neq \emptyset$ and thus $P(k) \neq \emptyset$. So the torsor $P \rightarrow$ Spec $k$ is trivial, and hence so is $E \rightarrow \operatorname{Spec} K$.

We observe that if $G$ is almost special then $\operatorname{ed}_{k} G=\operatorname{ed}_{k}(G ; p)$, where $p$ is the characteristic of $k$. In the following we give several examples of almost special group schemes. Most of them are connected and trigonalizable. We do not know whether all connected trigonalizable group scheme are almost special. For diagonalizable group scheme this is true (see Example 4.4). In the unipotent case this is open: in 4.9 we discuss what we consider to be a key example to clarify this question.

It is easy to give examples of infinitesimal almost special group schemes that are not trigonalizable: for example, if $\mathcal{G}$ is a special non-trigonalizable smooth group scheme (e.g. $\mathcal{G}=\mathrm{Gl}_{n}$ ), the kernel of its Frobenius ${ }_{\mathrm{F}} \mathcal{G}$ is almost special, as it follows from Theorem 1.2 and Lemma 2.1.

Finally we remark that if $G$ and $H$ are almost special then the product $G \times H$ is also almost special, and $\operatorname{ed}_{k}(G \times H)=\operatorname{ed}_{k} G+\operatorname{ed}_{k} H$.

The following strategy allows us to find some almost special group schemes. If an infinitesimal group scheme $G$ can be embedded in a special algebraic group scheme of dimension $n \stackrel{\text { def }}{=} \operatorname{dim}_{k} \operatorname{Lie}(G)$ then, using Theorem 1.2 and Lemma 2.1, we can conclude that the essential dimension is exactly $n$, i.e. $G$ is almost special. Here is an example when this happens. Later in 4.8 we will see another example in which this argument can not be applied.

Example 4.4. If $n$ is a positive integer, we denote by $W_{n}$ the group scheme of truncated Witt vectors of length $n$ (see [Ser79, Chapter 2, § 6], [Haz78, Chapter 3, $\S 1]$ and [DG70, Chapter $5, \S 1])$. Let $G$ be the group scheme

$$
\prod_{j=1}^{t} \mathrm{~F}^{m_{j}} W_{n_{j}} \times \prod_{i=1}^{s} \boldsymbol{\mu}_{p^{l_{i}}},
$$

where $\mathrm{F}^{m} W_{n}$ is the kernel of the iterated Frobenius $\mathrm{F}^{m}: W_{n} \rightarrow W_{n}$. Then $G$ is almost special, i.e.

$$
\operatorname{ed}_{k} G=s+\sum_{j=1}^{t} n_{j}=\operatorname{dim}_{k} \operatorname{Lie} G .
$$

Indeed, by the remark above, it is enough to remark that $G$ is a closed subgroup of $\prod_{j=1}^{t} W_{n_{j}} \times \mathbb{G}_{m}^{s}$, which is special.

Another class of examples is given by the following Corollary.

Corollary 4.5. Any trigonalizable group scheme $G$ of height $\leq 1$ and of finite type over $k$ is almost special.

Proof. If $G$ has order $p^{n}$ then, since $G$ has height $\leq 1$, the dimension of its Lie algebra is $n$. Therefore the result follows from Theorems 1.2 and 1.4.

As a consequence of Theorem 1.4 and Lemma 3.4 we prove the following result.

Corollary 4.6. Let

$$
1 \longrightarrow G_{1} \longrightarrow G \longrightarrow G_{2} \longrightarrow 1
$$


be an extension of group schemes over a field $k$ of characteristic $p>0$, with $G_{1}$ unipotent commutative of order $p^{n}$, then

$$
\operatorname{ed}_{k} G \leq n+\operatorname{ed}_{k} G_{2} .
$$

Proof. Let $P \rightarrow \operatorname{Spec} K$ be a $G$-torsor over an extension $K$ of $k$. Then, from Lemma 3.4, there exists an intermediate extension $k \subseteq E \subseteq K$ and a twisted form $\widetilde{G_{1}} \rightarrow \operatorname{Spec} E$ of $G_{1}$ over $E$, such that

$$
\operatorname{ed}_{k} P \leq \operatorname{ed}_{E} \widetilde{G_{1}}+\operatorname{ed}_{k} G_{2} .
$$

By Lemma 3.2, $\widetilde{G_{1}}$ is unipotent. Hence by Theorem 1.4 we have $\operatorname{ed}_{E} \widetilde{G_{1}} \leq n$, and the result follows.

Example 4.7. In addition to the hypotheses of Corollary 4.6, let us suppose that $G_{1}$ is of height $\leq 1, G_{2}$ is almost special and

$$
\operatorname{dim}_{k} \operatorname{Lie}(G)=\operatorname{dim}_{k} \operatorname{Lie}\left(G_{1}\right)+\operatorname{dim}_{k} \operatorname{Lie}\left(G_{2}\right) .
$$

From Corollary 4.6 and Theorem 1.2 it follows that

$$
\operatorname{ed}_{k} G=\operatorname{ed}_{k} G_{1}+\operatorname{ed}_{k} G_{2} .
$$

The hypothesis on the dimension of the Lie algebra of $G$ is satisfied, for instance, if the extension is split.

Example 4.8. We do not have examples of trigonalizable group schemes that are not almost special. If such an example exists a candidate should be the following.

Let us consider the kernel $G_{r, m, n}$ of the morphism $\mathrm{F}^{m}+V^{r}: \mathrm{W}_{n} \rightarrow \mathrm{W}_{n}$, where $V$ is the Verschiebung (called "shift" in [Ser79] and "décalage" in [DG70]), $m>0$ and $0<r<n$. This is a group scheme of order $p^{m n}$. The dimension of its Lie algebra is $r$, and it is embedded in a special group scheme of dimension $n$, therefore $r \leq \operatorname{ed}_{k} G_{r, m, n} \leq n$.

Now consider the case $r=1$. Then we will prove that $G_{1, m, n}$ can not be embedded in any special algebraic group scheme $\mathcal{G}$ of dimension 1 , so we can not use an argument as in the Example 4.4 to conclude that $\mathrm{ed}_{k} G_{1, m, n}=1$. To prove this fact we can clearly suppose that $k$ is algebraically closed. Now let us suppose that such a group $\mathcal{G}$ exists. First of all we can suppose it is connected. Secondly we observe that it should be unipotent. Indeed, since it is special it should be smooth (see Theorem 1.2) and affine ([Ser58, Theorem 1]); therefore, if it was not unipotent, from [DG70, Proposition IV $\S 2,3.11]$ we conclude that it contains a subgroup scheme isomorphic to $\mathbb{G}_{m}$. This implies that the kernel of Frobenius, ${ }_{\mathrm{F}} \mathcal{G}$, is not unipotent, since it contains a subgroup scheme isomorphic to $\boldsymbol{\mu}_{p}$. But since the Lie algebra of $G_{1, m, n}$ is equal to the Lie algebra of $\mathcal{G}$ we have that ${ }_{\mathrm{F}} \mathcal{G}={ }_{\mathrm{F}} G_{1, m, n}$, which is a contradiction since $G_{1, m, n}$ is unipotent. But any unipotent smooth and connected group scheme of dimension 1 over a perfect field is isomorphic to $\mathbb{G}_{a}$ ([DG70, Corollary IV $\S 2,2.10]$ ). And $G_{1, m, n}$ is not a subgroup scheme of $\mathbb{G}_{a}$.

This example is also interesting since, if $k$ is perfect, the group scheme $G_{1,1,2}$ is the $p$-torsion group scheme of a supersingular elliptic curve (i.e., an elliptic curve with $p$-rank equal to zero). The $p^{n}$-torsion group scheme of the same curve has a decomposition series with quotients isomorphic to $G_{1,1,2}$.

Example 4.9. In [Led04], Ledet conjectured that the essential dimension of $\mathbb{Z} / p^{n} \mathbb{Z}$ over a field $k$ of characteristic $p$ is $n$. The conjecture has been proved (easily) for $n \leq 2$. Since $\mathbb{Z} / p^{n} \mathbb{Z}$ is contained in the group scheme of Witt vectors of dimension 
$n, \mathrm{~W}_{n}$, and this group is special then $\operatorname{ed}_{k}\left(\mathbb{Z} / p^{n} \mathbb{Z}\right) \leq n$. The same result also follows from Theorem 1.4, which for constant $p$-group schemes was already known. The open problem (for $n>2$ ) is to prove the opposite inequality. We remark that if this conjecture is true over an algebraic closure $\bar{k}$ of $k$ then $\operatorname{ed}_{k} G$ is equal to $n$ for any twisted form $G$ of $\mathbb{Z} / p^{n} \mathbb{Z}$. Indeed such a group scheme would be unipotent (see Lemma 3.1) so, by the Theorem 1.4, its essential dimension is smaller or equal to $n$. On the other hand it is at least $n$, since the essential dimension does not increase by base change (see [BF03, Proposition 1.5]).

\section{REFERENCES}

[BF03] Grégory Berhuy and Giordano Favi, Essential dimension: a functorial point of view (after A. Merkurjev), Doc. Math. 8 (2003), 279-330 (electronic).

[BR97] Joe Buhler and Zinovy Reichstein, On the essential dimension of a finite group, Compositio Math. 106 (1997), no. 2, 159-179.

[Bro07] Patrick Brosnan, The essential dimension of a g-dimensional complex abelian variety is 2g, Transformation Groups 12 (2007), no. 3, 437-441.

[BRV09] Patrick Brosnan, Zinovy Reichstein, and Angelo Vistoli, Essential dimension of moduli of curves and other algebraic stacks, arXiv:0907.0924, to appear in Journal of European Mathematical Society, 2009.

[BRV10] , Essential dimension, spinor groups, and quadratic forms, Ann. of Math. (2) 171 (2010), no. 1, 533-544.

[BS08] Patrick Brosnan and Ramesh Sreekantan, Essential dimension of abelian varieties over number fields, C. R. Math. Acad. Sci. Paris 346 (2008), no. 7-8, 417-420.

[CS06] Vladimir Chernousov and Jean-Pierre Serre, Lower bounds for essential dimensions via orthogonal representations, J. Algebra 305 (2006), no. 2, 1055-1070.

[DG70] Michel Demazure and Pierre Gabriel, Groupes algébriques. Tome I: Géométrie algébrique, généralités, groupes commutatifs, Masson \& Cie, Éditeur, Paris, 1970, Avec un appendice it Corps de classes local par Michiel Hazewinkel.

[Flo07] Mathieu Florence, On the essential dimension of cyclic p-groups, Inventiones mathematicae 171 (2007), 175-189.

[Gir71] Jean Giraud, Cohomologie non abélienne, Springer-Verlag, Berlin, 1971, Die Grundlehren der mathematischen Wissenschaften, Band 179.

[GMS03] Skip Garibaldi, Alexander Merkurjev, and Jean-Pierre Serre, Cohomological invariants in galois cohomology, University Lecture Series, vol. 28, American Mathematical Society, 2003

[GR09] Philippe Gille and Zinovy Reichstein, A lower bound on the essential dimension of a connected linear group, Comment. Math. Helv. 84 (2009), no. 1, 189-212.

[Gro58] Alexander Grothendieck, Torsion homologique et sections rationnelles, Anneaux de Chow et Applications, Séminaire Claude Chevalley, 1958, expoeé n. 5.

[Haz78] Michiel Hazewinkel, Formal groups and applications, Pure and Applied Mathematics, vol. 78, Academic Press Inc. [Harcourt Brace Jovanovich Publishers], New York, 1978.

[KM08] Nikita A. Karpenko and Alexander S. Merkurjev, Essential dimension of finite pgroups, Inventiones Mathematicae 172 (2008), no. 3, 491-508.

[Led04] Arne Ledet, On the essential dimension of p-groups, Galois Theory and Modular Forms, Dev. Math., vol. 11, Kluwer Acad. Publ., 2004, pp. 159-172.

[Liu02] Qing Liu, Algebraic geometry and arithmetic curves, Oxford Graduate Texts in Mathematics, vol. 6, Oxford University Press, 2002.

[LMMR09] Roland Lötscher, Mark MacDonald, Aurel Meyer, and Zinovy Reichstein, Essential dimension of algebraic tori, http://www.math.ubc.ca/ reichst/edtorishort5-11-10. pdf, 2009.

[Mer09] Alexander S. Merkurjev, Essential dimension, Quadratic forms - algebra, arithmetic, and geometry, Contemp. Math., vol. 493, Amer. Math. Soc., Providence, RI, 2009, pp. 299-325.

[Mil80] James S. Milne, Étale cohomology, Princeton Mathematical Series, vol. 33, Princeton University Press, Princeton, N.J., 1980. 
[Rei00] Zinovy Reichstein, On the notion of essential dimension for algebraic groups, Transformation Groups 5 (2000), no. 3, 265-304.

[RY00] Zinovy Reichstein and Boris Youssin, Essential dimensions of algebraic groups and a resolution theorem for $G$-varieties, Canad. J. Math. 52 (2000), no. 5, 1018-1056, With an appendix by János Kollár and Endre Szabó.

[Ser58] Jean-Pierre Serre, Espaces fibres algébriques, Anneaux de Chow et Applications, Séminaire Claude Chevalley, 1958, exposé n. 1.

[Ser79] , Local fields, Graduate Texts in Mathematics, vol. 67, Springer-Verlag, New York, 1979.

[Ste65] Robert Steinberg, Regular elements of semisimple algebraic groups, Inst. Hautes Études Sci. Publ. Math. (1965), no. 25, 49-80.

Scuola Normale Superiore, Piazza dei Cavalieri 7, 56126 Pisa, Italy

E-mail address, Tossici: dajano.tossici@sns.it

E-mail address, Vistoli: angelo.vistoli@sns.it 